\title{
Self-resonant modes in Josephson junctions with a phase discontinuity
}

\author{
C. Nappi, M. Adamo, and E. Sarnelli \\ Istituto di Cibernetica «E. Caianiello» del C.N.R. I-80078, Pozzuoli, Napoli, Italy \\ E-mail: c.nappi@cib.na.cnr.it \\ E. Goldobin
}

Physikalisches Institut-Experimentalphysik II, Universität Tübingen, Auf der Morgenstelle 14, D-72076, Tübingen, Germany

Received April 9, 2010

\begin{abstract}
We extend the theory of self-resonances in short Josephson junctions to the case of a piecewise constant critical current density and a $\kappa$ discontinuity of the Josephson phase. We calculate the amplitudes of self-resonances as a function of applied magnetic field by using an extension of the approach introduced by Kulik for conventional Josephson junctions (I.O. Kulik, JETP Lett. 2, 84 (1965)). The presented theory agrees with existing experiments on superconducting-insulator-ferromagnet-superconductor $0-\pi$ Josephson junctions. The results are relevant for the characterization of all modern $0-\pi$ junctions as well as $0-\kappa$ junctions with artificially created phase discontinuity: high-temperature grain boundary junctions, junctions with ferromagnetic barrier and the junction with current injectors.
\end{abstract}

PACS: 85.25.Cp Josephson devices;

61.72.Mm Grain and twin boundaries;

85.25.-j Superconducting devices.

Keywords: critical current density, plasma frequency, Fiske steps.

\section{Introduction}

Kulik's explanation [1] of Fiske steps [2] in short Josephson junctions (JJs) has revealed a key to the understanding of important features on the $I-V$ characteristic (IVC) of a JJ.

The theory accounts for the nonlinear coupling of currents and fields in the Josephson junction and allows to predict the IVC of the JJ when magnetic field is applied. In particular, the dependence of the amplitudes of Fiske steps versus the applied magnetic field can be predicted [3]. In the course of the years this model has been generalized to more complex Josephson structures: two-dimensional JJs [4], annular JJs [5], SQUIDs or arrays of JJs [6], etc.

A well documented [7] early generalization of the $\mathrm{Ku}$ lik's model, is the case in which the critical current density is nonuniform, i.e., smooth or even steplike function of the coordinate $[8,9]$.

The steplike case has been recently revisited in context of the novel $0-\pi$ JJs [10], where the critical current density $j_{c}$ in some parts of JJs may become negative, which corresponds to an additional Josephson phase shift of $\pi$ [11].
While in conventional $\mathrm{JJ}$ with $j_{c}>0$ the ground state corresponding to the minimum of the Josephson energy density

$$
U(\varphi)=-\frac{\Phi_{0} j_{c}}{2 \pi} \cos \varphi
$$

is $\varphi=0$, the ground state of the JJ with $j_{c}<0$ is $\varphi=\pi$. Thus, in a sufficiently long $0-\pi \mathrm{JJ}$, the ground state Josephson phase $\varphi(x)$ bends from 0 to $\pi$ in a $\lambda_{J}$ vicinity of a $0-\pi$ boundary, where $\lambda_{J}$ is the Josephson penetration depth. As a result, a spontaneous localized magnetic flux $\Phi \leq \Phi_{0} / 2$, where $\Phi_{0}=2.07 \cdot 10^{-15} \mathrm{~Wb}$ is a magnetic flux quantum, appears at a $0-\pi$ boundary. For very long $0-\pi \mathrm{JJ}, \quad \Phi \rightarrow \Phi_{0} / 2$ and one speaks about semifluxons pinned at $0-\pi$ boundary [12-14]. However, in this paper we focus on short JJs with $L \lesssim 4 \lambda_{J}$.

The possibility to make experiments with novel JJs in which $0-\pi$ phase jumps may be naturally observed or artificially created (like in a specially oriented submicron HTS grain boundary junctions with only several facets or in superconductor-insulator-ferromagnet-superconductor SIFS) 
JJs or JJs with current injectors) makes the self-resonances argument of renewed topical interest.

In this paper we revisit the theory of self-resonances in $0-\pi$ junctions [10] and generalize it to the case of an arbitrary $\kappa$ discontinuity, relevant in the context of JJs with artificial discontinuity [15], and to the case of unequal critical current densities $j_{1} \neq j_{2}$ in two parts of a JJ, relevant in the context of SIFS JJs [16]. Finally we compare the presented model with the experiment results obtained using $0-\pi$ SIFS JJs [17].

\section{Josephson junctions with a phase discontinuity}

Let us consider a Josephson junction, extending from 0 to $L$ along the $y$ coordinate, with two adjacent regions $[0, l]$ and $[l, L]$, characterized by two different critical current densities, $j_{1}$ and $j_{2}$. We also assume that passing from the first region to the second region a jump in the phase difference occurs, so that the value of the overall phase difference changes abruptly from the value $\varphi$ to the value $\varphi+\kappa$ at $y=l$. Two possible realizations of such a physical system, in the specific case of a $0-\pi$ phase jump, are schematically shown in Fig. 1 together with the coordinate system used in this work. Figure 1,a represents a two facet section of a grain boundary junction based on high critical temperature superconducting films and Fig. 1,b a SIFS overlap type junction.

The supercurrent in these system is given by

$$
j(y)=\left\{\begin{array}{ll}
j_{1} \sin \varphi & \text { if } 0<y<l \\
j_{2} \sin (\varphi+\kappa) & \text { if } l<y<L
\end{array} .\right.
$$
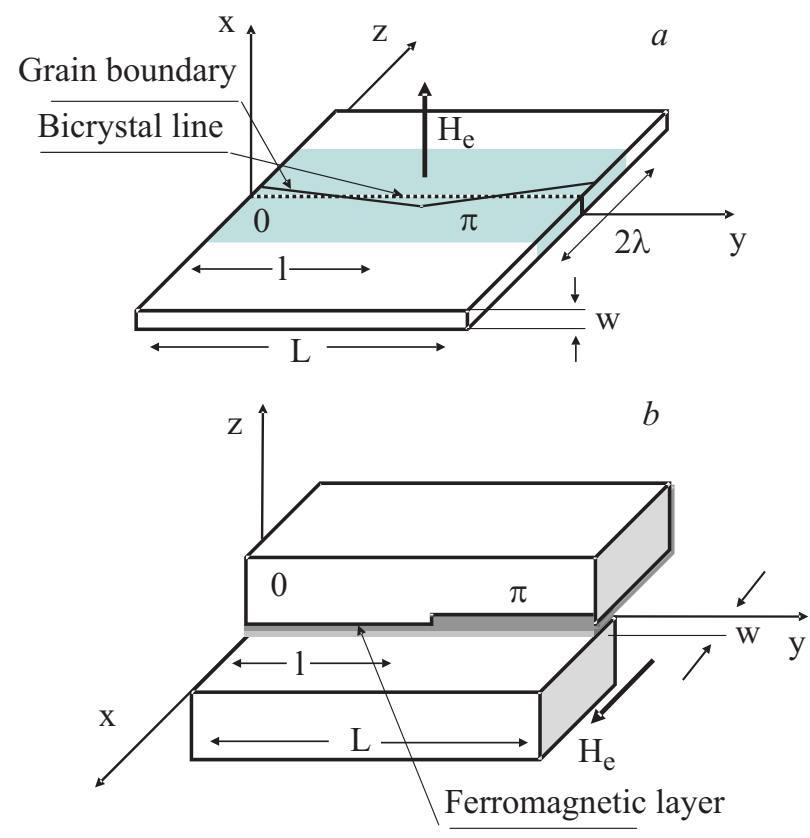

Fig. 1. Schematic representation of investigated JJs. (a) A grain boundary junction. (b) A SIFS overlap junction.
In the case of a $\pi$ phase jump, $\sin (\varphi+\pi)=-\sin \varphi$, and, thus, the $\pi$-jump is equivalent to a sign change in the critical current density. The above schematization is sufficiently general to cover all existing Josephson systems relevant for this work: (i) HTS $0-\pi$ grain boundary junctions $[10,18,19]$ where $\kappa=\pi$ and $j_{1}=j_{2}$; (ii) JJs with ferromagnetic barrier [16,17] where $\kappa=0, j_{1} \neq j_{2}$; and (iii) $0-\kappa \quad \mathrm{JJ} \quad j_{1}=j_{2}$ and with artificial and tunable $\kappa-$ discontinuity of the phase created by current injectors (not shown in Fig. 1). Actually the first case is equivalent to the second one with $j_{1}=-j_{2}$.

Therefore, below we assume that $j_{1}>0$ and $j_{2}>0$ and the possible different sign of $j_{c}$, if necessary, will be substituted by additional phase shift of $\pi$ in the corresponding part of the JJ.

\section{Calculations}

The equation for the phase difference $\varphi(y, t)$ in the reference frame shown in Fig. 1 and with the above assumptions may be written $(0<y<L)$

$$
\begin{gathered}
\left\langle\lambda_{j}\right\rangle^{2} \frac{\partial^{2} \varphi}{\partial y^{2}}-\frac{1}{\left\langle\omega_{p}\right\rangle^{2}} \frac{\partial^{2} \varphi}{\partial t^{2}}-\frac{1}{R C\left\langle\omega_{p}\right\rangle^{2}} \frac{\partial \varphi}{\partial t}= \\
=\frac{j_{c}(y)}{\left\langle j_{c}\right\rangle} \sin [\varphi+\kappa \theta(y-l)]-\gamma
\end{gathered}
$$

where we have introduced the specific normal resistance $R$ and capacitance $C$ of the junction. Function $\theta(y)$ is a Heaviside step function, $\gamma=j_{e} /\left\langle j_{c}\right\rangle$ is the normalized external bias current density. $j_{c}$ is the critical current density that, in turn is assumed a discontinuous function of the position as well

$$
j_{c}(y)=\left\{\begin{array}{ll}
j_{1} & \text { if } 0<y<l \\
j_{2} & \text { if } l<y<L
\end{array} .\right.
$$

Accordingly we have introduced the average critical current density

$$
\left\langle j_{c}\right\rangle=\frac{j_{1} l+j_{2}(L-l)}{L},
$$

the average Josephson penetration depth

$$
\left\langle\lambda_{j}\right\rangle=\sqrt{\frac{\Phi_{0}}{2 \pi \mu_{0}\left\langle j_{c}\right\rangle d_{\mathrm{eff}}}},
$$

and the average plasma frequency

$$
\left\langle\omega_{p}\right\rangle=\sqrt{\frac{2 \pi\left\langle j_{c}\right\rangle}{\Phi_{0} C}},
$$

respectively. All lengths and time variables are from now on normalized to $\left\langle\lambda_{j}\right\rangle$ and to the inverse average plasma frequency $\left\langle\omega_{p}\right\rangle$, respectively, so that Eq. (3) becomes 


$$
\varphi_{y y}-\varphi_{t t}-\alpha \varphi_{t}=\frac{j_{c}(y)}{\left\langle j_{c}\right\rangle} \sin [\varphi+\kappa \theta(y-l)]-\gamma,
$$

where $\alpha=1 /\left\langle\omega_{p}\right\rangle R C$ is the dimensionless damping coefficient.

The appropriate boundary conditions to Eq. (5) are

$$
\varphi_{y}(0, t)=\varphi_{y}(L, t)=-h,
$$

where $h=H_{e} /\left\langle\lambda_{j}\right\rangle\left\langle j_{c}\right\rangle$ is the normalized external magnetic field aligned along the positive direction of the $x$ axis. The quantity $h$ determines the spatial modulation of the phase $\varphi(y)$ in the presence of the magnetic field $H_{e}$, i.e., $\varphi(y)=-h y$.

The IVC can be derived starting from

$$
j=j_{c}(y) \sin [\varphi(y, t)+\kappa \theta(y-l)],
$$

where $\varphi(y, t)$ is a continuous function. The Kulik approximation consists of writing the unknown part of the phase $\varphi$ as a sum of two terms $\varphi=\varphi_{0}+\varphi_{1}$, where $\varphi_{1} \ll \varphi_{0}$ and $v=\left(\Phi_{0} / 2 \pi\right) d \varphi_{1} / d t$ is the perturbation to the steady voltage $V$ :

$$
\varphi(y, t)=\varphi_{0}(y, t)+\varphi_{1}(y, t)=\omega t-h y+\varphi_{1}(y, t) .
$$

Here $\omega=\left(2 \pi V / \Phi_{0}\right) /\left\langle\omega_{p}\right\rangle$ is the normalized Josephson frequency corresponding to the fixed voltage $V$ applied between electrodes of the JJ. The linear equation providing $\varphi_{1}$ is derived by substituting Eq. (7) into Eq. (5) and then neglecting the perturbation $\varphi_{1}$ in the argument of sine [20]:

$\varphi_{1 y y}-\varphi_{1 t t}-\alpha \varphi_{1 t}= \begin{cases}\left(j_{1} /\left\langle j_{c}\right\rangle\right) \sin (\omega t-h y) & \text { if } 0<y<l \\ \left(j_{2} /\left\langle j_{c}\right\rangle\right) \sin (\omega t-h y+\kappa) & \text { if } l<y<L\end{cases}$

Equation (8) is completed with the boundary conditions derived directly from Eq. (6) and expressing perfect edge, reflectivity,

$$
\varphi_{1 y}(0, t)=\varphi_{1 y}(L, t)=0 .
$$

Following a standard procedure $[1,7]$, we seek $\varphi_{1}(y, t)$ in the form

$$
\varphi_{1}(y, t)=\sum_{n=1}^{\infty} a_{n} \cos \left(\frac{n \pi y}{L}\right) \cos \omega t+\sum_{n=1}^{\infty} b_{n} \cos \left(\frac{n \pi y}{L}\right) \sin \omega t
$$

The coefficients are straightforwardly obtained by substituting Eq. (10) in Eq. (8). They are given by

$$
a_{n}=-\frac{1}{\omega^{2}} \frac{\left[\left(1-\frac{\omega_{n}^{2}}{\omega^{2}}\right) B_{n}-\frac{\alpha}{\omega} C_{n}\right]}{\left[\left(1-\frac{\omega_{n}^{2}}{\omega^{2}}\right)^{2}+\frac{\alpha^{2}}{\omega^{2}}\right]},
$$

$$
b_{n}=\frac{1}{\omega^{2}} \frac{\left[\left(1-\frac{\omega_{n}^{2}}{\omega^{2}}\right) C_{n}+\frac{\alpha}{\omega} B_{n}\right]}{\left[\left(1-\frac{\omega_{n}^{2}}{\omega^{2}}\right)^{2}+\frac{\alpha^{2}}{\omega^{2}}\right]},
$$

where

$$
\begin{gathered}
B_{n}=\frac{2}{L} \frac{j_{1}}{\left\langle j_{c}\right\rangle} \int_{0}^{l} \sin (h y) \cos \left(\frac{n \pi y}{L}\right) d y+ \\
+\frac{2}{L} \frac{j_{2}}{\left\langle j_{c}\right\rangle} \int_{l}^{L} \sin (h y-\kappa) \cos \left(\frac{n \pi y}{L}\right) d y, \\
C_{n}=\frac{2}{L} \frac{j_{1}}{\left\langle j_{c}\right\rangle} \int_{0}^{l} \cos (h y) \cos \left(\frac{n \pi y}{L}\right) d y+ \\
+\frac{2}{L} \frac{j_{2}}{\left\langle j_{c}\right\rangle} \int_{l}^{L} \cos (h y-\kappa) \cos \left(\frac{n \pi y}{L}\right) d y,
\end{gathered}
$$

and

$$
\omega_{n}=n \pi / L, \quad n=1,2, \ldots
$$

are the normalized resonance frequencies. Note that this result for $\omega_{n}$ is the same found in the case of a conventional JJ with uniform critical current density. Thus the phase jumps have no influence on the distribution of the resonance frequencies. The Josephson current density is given by

$$
j=j_{c}(y) \sin \left[\varphi_{0}+\varphi_{1}+\kappa \theta(y-l)\right] .
$$

This current is frequency modulated and contains a nonzero dc term which can be extracted by using a time,

$$
\begin{aligned}
\overline{(\ldots)}= & \frac{1}{2 \pi} \int_{0}^{2 \pi} \ldots d(\omega t), \text { and space averaging } \\
& J_{d c} \approx \frac{1}{L} \int_{0}^{L} j_{c}(y) \overline{\varphi_{1} \cos [\omega t-h y+\kappa \theta(y-l)]} d y .
\end{aligned}
$$

The result is

$$
J_{d c}=\frac{\left\langle j_{c}\right\rangle}{4 \omega^{2}} \sum_{n=1}^{\infty} \frac{\alpha / \omega}{\alpha^{2} / \omega^{2}+\left(1-\omega_{n}^{2} / \omega^{2}\right)^{2}}\left[B_{n}^{2}+C_{n}^{2}\right]
$$

At a fixed value of the external magnetic field this expression is a sum of many current peaks centered at the voltages $V_{n}=\omega_{n}\left\langle\omega_{p}\right\rangle \Phi_{0} / 2 \pi$. We introduce the external flux $\Phi=\mu_{0} H_{e} \Lambda L$, where $\Lambda \approx 2 \lambda_{L}$ is the effective penetration depth of the magnetic field into the junction and $\lambda_{L}$ is the London penetration depth, so that $h L=$ $=2 \pi \Phi / \Phi_{0}=2 \pi \phi$, where $\phi$ is the normalized applied 
flux (frustration). We define $F_{n}^{2}(\phi)=B_{n}^{2}+C_{n}^{2}$ and calculate the normalized maximum amplitude at $\omega=\omega_{n}$ (15) of the $n$th step as

$$
\frac{J_{n}^{M}(\phi)}{\left\langle j_{c}\right\rangle}=\frac{L^{2}}{4} \frac{Q_{n}}{n^{2} \pi^{2}} F_{n}^{2}(\phi),
$$

where the quality factor $Q_{n}=\omega_{n} / \alpha$ has been introduced. In particular in zero field $(\phi=0)$

$$
F_{n}^{2}(0)=\frac{4\left[\left(1+s^{2}-2 s \cos \kappa\right) \sin ^{2}\left(n \pi \frac{l}{L}\right)\right]}{\left[s \frac{l}{L}+1-\frac{l}{L}\right]^{2} n^{2} \pi^{2}},
$$

where $s=j_{1} / j_{2}$. When $s=1$ and the singularity is in the center $(l / L=0.5)$, Eq. (19) gives

$$
F_{n}(0)^{2}= \begin{cases}8(1-\cos \kappa) / n^{2} \pi^{2}, & n=1,3, \ldots \\ 0, & n=2,4, \ldots\end{cases}
$$

\section{Results}

Figures 2 show the dependence of the Fiske step amplitude $J_{n}^{M}(\phi)$, calculated using Eq. (18), for three different values of $\kappa(\kappa=0, \pi / 2, \pi)$ and $n=1,2,3$, respectively. Calculations have been carried out considering a junction with a normalized length $L=2$ and a quality factor $Q=1$ independent from the order $n$ of the self-resonance.

These figures illustrate a general feature of these kind of curves (we limit this discussion to the case $j_{1}=j_{2}$ ). Each of them is, generally speaking, asymmetric with respect to magnetic field inversion, i.e., $J_{n}^{M}(\phi, \kappa) \neq J_{n}^{M}(-\phi, \kappa)$ for generic values $0<\kappa<2 \pi$. This can be appreciated in Figs. $2, a-c$, for the case $\kappa=\pi / 2$ (dashed lines). Only with $\kappa=0(2 \pi)$ or $\kappa=\pi$, the resulting curves are symmetric, i.e., $J_{n}^{M}(\phi, \kappa)=J_{n}^{M}(-\phi, \kappa)$ (solid line and dot-dashed line in Figs. 2,a-c). Moreover couple of curves corresponding to two complementary values of $\kappa$, i.e., $\kappa$ and $2 \pi-\kappa$, appear symmetric with respect to the magnetic field inversion, i.e., $J_{n}^{M}(\phi, \kappa)=J_{n}^{M}(-\phi, 2 \pi-\kappa)$. This, for instance, would be the case of the curves $J_{1}^{M}(\phi, 3 \pi / 2)$ (not shown for clarity in Fig. 2,a) which would be symmetric of $J_{1}^{M}(\phi, \pi / 2)$, i.e., $J_{1}^{M}(-\phi, 3 \pi / 2)=J_{1}^{M}(\phi, \pi / 2)$.

Looking at the first Fiske step (Fig. 2,a) one sees that at $\kappa \rightarrow 0 \quad($ or $\kappa \rightarrow 2 \pi)$ the step is absent at zero field, and obtains the largest amplitude at $\phi \sim 1$. Contrary, for $\kappa=\pi$, the first Fiske step is the most developed at zero applied field. Therefore, in the literature, this step is sometimes called half-integer zero field step $[17,22,23]$. For $0-\pi$ junction of moderate length it can be explained as a permanent flipping of semifluxon accompanied by the transfer of one flux quantum $\Phi_{0}$ through one of the JJ ends [21].

The third and, actually, all futher odd Fiske steps have similar behavior. For $\kappa \rightarrow 0$ or $\kappa \rightarrow 2 \pi$ they are not visible at zero field and appear only when the field $(\phi \sim 1.8$ for $n=3$ ) is applied to the junction. For $\kappa=\pi$, they are visible
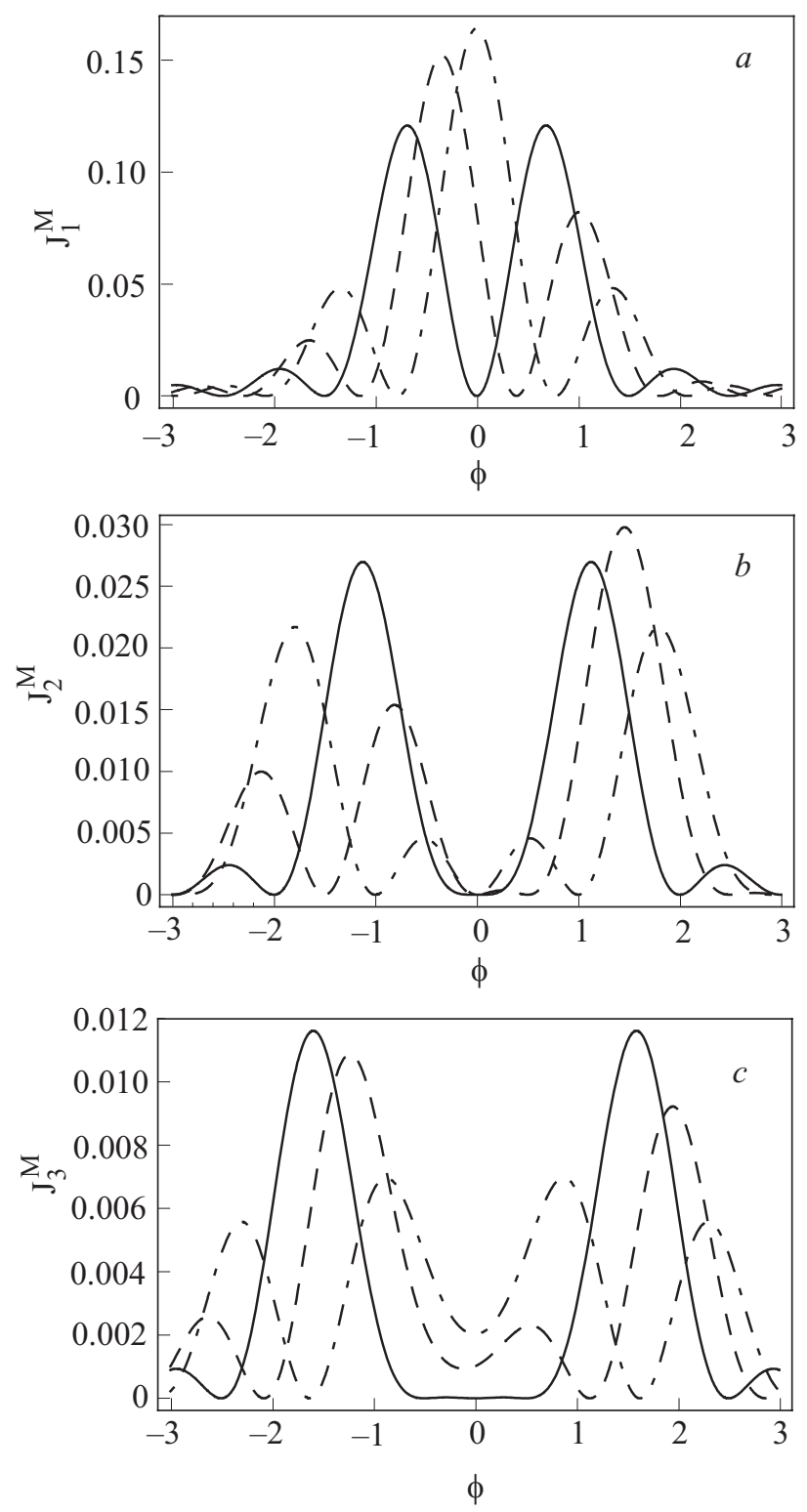

Fig. 2. Magnetic field dependence of the normalized amplitudes $J_{n}^{M} /\left\langle j_{c}\right\rangle$ of the first Fiske step $(n=1)(a)$, second Fiske step $(n=2)(b)$, and third Fiske step $(n=3)(c)$ in a $0-\kappa \mathrm{JJ}$ with $L=2$ $, l / L=0.5, \quad j_{1} / j_{2}=1, Q_{n}=1$, and $\kappa=0 \quad(2 \pi) \quad$ (solid line), $\pi / 2$ (dashed line), $\pi$ (dot-dashed line).

also in zero field. However, opposite to the $n=1$ case, they have a minimum of finite amplitude rather than a maximum at $\phi=0$.

The amplitudes of even Fiske steps, in particular of the second one shown in Fig. 2, a always vanish at $\phi=0$ regardless of $\kappa$ value, in agreement with Eq. (20). They also have smaller amplitude than the odd Fiske steps with comparable $n$.

\section{Comparison with experimental results}

In this section we compare our theory with experiments reported in Ref. 17, which were accomplished using SIFS $0-\pi \mathrm{JJ}$, similar to the one schematically shown in Fig. 1,b, 
cf. Fig. 1 of Ref. 17. Up to now there are no published experimental data on the general case of Fiske steps in $0-\kappa$ JJs with $\kappa \neq(0, \pi)$.

The JJs investigated in Ref. 17 had the length $L=330 \mu \mathrm{m}$, width $w=30 \mu \mathrm{m}$. The critical current density at $T=4.2 \mathrm{~K}$ was estimated as $j_{c} \approx 1.8 \mathrm{~A} / \mathrm{cm}^{2}$. Taking into account the idle region (width $50 \mu \mathrm{m}$ ) correction, we estimate the normalized JJ length as $L / \lambda_{J} \approx 0.67$ [17].

In Fig. 5 of Ref. 17 the families of IVCs taken at different magnetic fields are shown. One can see Fiske steps which are equally spaced in spite of the presence of a phase discontinuity, in accordance with the theory presented here.

The height $I_{n}^{M}$ of each step including the critical current $(n=0)$ is measured as a function of magnetic field $H_{e}$. The results are presented in Fig. 5 of Ref. 17. One can see that the critical current $I_{c}(H)$ exibits a cusplike minimum at $H_{e}=0$. This is the result of cancelation of supercurrents flowing in different parts of the JJ. At the same time the amplitude of the first Fiske step $I_{1}^{M}\left(H_{e}\right)$ has a maximum at $H_{e}=0$. This behavior is opposite to the one observed in conventional $\mathrm{JJ}$, where $I_{c}\left(H_{e}\right)$ has a maximum and $I_{1}^{M}\left(H_{e}\right)$ has a minimum at zero applied magnetic field.

Earlier it was suggested to interpret the 1st Fiske step in a $0-\pi \mathrm{JJ}$ as a «semi-integer zero field step». For the first time the semi-integer zero field step was observed on numerically simulated IVC of a $0-\pi$ of moderate length [22]. The dynamics of magnetic flux on this step was described in details for the case of moderately long $0-\pi \mathrm{JJs}$ [15] by considering the flipping of semifluxon (and its image) under the action of the Lorenz force [21]. Later it became clear that one can obtain this step in the framework of the Kulik's Fiske step theory, which works well in the limit of short JJ [10].

In agreement with the earlier predictions [22,23], the theory provides the following conclusions. In the presence of an externally applied magnetic field, resonances appear at all positions $\omega_{n}$ given by Eq. (15), similarly to a conventional junction. However, in contrast with conventional behavior, when the magnetic field is equal to zero, the resonances with odd $n$ are visible. In a $0-\pi \mathrm{JJ}$ of moderate length the positions of resonances remain the same, and the Fiske step with odd $n$ may be interpreted as half-integer zero field steps [17].

\section{Summary}

In this contribution, we have shown how to extend the Kulik theory to the case of self-resonances that appear in novel $0-\pi$ Josephson junctions. The extended model takes into account different critical current densities $j_{1} \neq j_{2}$ in two regions as well as arbitrary phase shift $\kappa$ between them. This extended Kulik's formalism is well applicable to such new systems and describes experimental data (at least for the case of $0-\pi \mathrm{JJs}$ ) rather well.

\section{Acknowledgments}

This work has been partially supported by EU STREP project MIDAS, «Macroscopic Interference Devices for Atomic and Solid State Physics: Quantum Control of Supercurrents».

1. I.O. Kulik, JETP Lett. 2, 84 (1965).

2. M.D. Fiske, Rev. Mod. Phys. 36, 221 (1964).

3. Fiske steps are often indicated as «self-resonances», because they are driven by the self-generated ac current in the JJ.

4. M.A.H. Nerenberg, P.A. Forsyth, Jr., and J.A. Blackburn, $J$. Appl. Phys. 17, 2171 (1978).

5. C. Nappi, R. Cristiano, and M.P. Lisitskii, Phys. Rev. B58, 11685 (1998).

6. B. Chesca and R. Kleiner, Physica C350, 180 (2001).

7. A. Barone and G. Paternó, Physics and Applications of the Josephson Effect, Wiley, NY (1982).

8. M. Russo and R. Vaglio, Phys. Rev. B17, 2171 (1978).

9. G. Paternó, Josephson Effect, Achievements and Trends, World Scientific, Singapore (1986), p. 139.

10. C. Nappi, E. Sarnelli, M. Adamo, and M.A. Navacerrada, Phys. Rev. B74, 144504 (2006).

11. H. Hilgenkamp, J. Mannhart, and B. Mayer, Phys. Rev. B53, 14586 (1996).

12. L.N. Bulaevskii, V.V. Kuzii, and A.A. Sobyanin, Solid State Commun. 25, 1053 (1978).

13. J.H. Xu, J.H. Miller, and C.S. Ting, Phys. Rev. B51, 11958 (1995).

14. E. Goldobin, D. Koelle, and R. Kleiner, Phys. Rev. B66, 100508(R) (2002).

15. E. Goldobin, A. Sterck, T. Gaber, D. Koelle, and R. Kleiner, Phys. Rev. Lett. 92, 057005 (2004).

16. M. Weides, M. Kemmler, E. Goldobin, H. Kohlstedt, R. Waser, D. Koelle, and R. Kleiner, Phys. Rev. Lett. 97, 247001 (2006).

17. J. Pfeiffer, M. Kemmler, D. Koelle, R. Kleiner, E. Goldobin, M. Weides, A.K. Feofanov, J. Lisenfeld, and A.V. Ustinov, Phys. Rev. B77, 214506 (2008).

18. D.A. Wollman, D.J. Van Harlingen, W.C. Lee, D.M. Ginsberg, and A.J. Leggett, Phys. Rev. Lett. 73, 1872 (1994).

19. H.J.H. Smilde, Ariando, D.H.A. Blank, G.J. Gerritsma, H. Hilgenkamp, and H. Rogalla, Phys. Rev. Lett. 88, 057004 (2002).

20. The zero-th order approximation gives the quasiparticle dissipative contribution to the current-voltage characteristic, $\gamma=\alpha \omega$, or in the present units $j=V / R$.

21. E. Goldobin, D. Koelle, and R. Kleiner, Phys. Rev. B67, 224515 (2003).

22. N. Stefanakis, Phys. Rev. B66, 214524 (2002).

23. N. Lazarides, Phys. Rev. B69, 212501 (2004). 